\title{
La enseñanza en la educación superior requiere aplicar estrategias de logro por los docentes, en la búsqueda del conocimiento continuo del alumno
}

\author{
Teaching in higher education requires applying strategies \\ of achievement by teachers, in the search of the continuous \\ knowledge of the pupil
}

\begin{abstract}
RESUMEN
La presente investigación se encuentra orientada a buscar estrategias que generalmente contribuyan a la calidad en los resultados de la labor docente, cuya responsabilidad es de contribuir con los conocimientos continuos de los alumnos, que se forman en la Educación Superior.

Del mismo modo, se incide en cuanto a la eficiencia en la labor docente, así como en el fomentar, inculcar, y defender el desarrollo de criterio del alumno; el resaltar que las instituciones educativas, como el gobierno central deben obligarse a establecer normas y políticas, y otras que demanden responsabilidad, innovación en los docentes de la Educación Superior.

Palabras claves: Enseñanza; conocimiento; estrategias.
\end{abstract}

\begin{abstract}
The present investigation is oriented to look for strategies that generally contribute to the quality in the results of the teaching work, whose responsibility is to contribute with the continuous knowledge of the students that are formed in Higher Education.

In the same way it affects the efficiency in the teaching work as well as the encouragement, inculcate, and defend the development of the student's criteria; to emphasize that educational institutions, such as the central government must be obliged to establish norms and policies, and others that demand responsibility, innovation in teachers of Higher Education.
\end{abstract}

Keywords: Teaching; knowledge; strategies.

\section{Félix Armando Rivera León ${ }^{1}$}

felix_arl@hotmail.com

\section{Jorge Luis Alarcon Cerna ${ }^{2}$}

jorge.alarcon@unmsm.edu.pe

Universidad Nacional Mayor de San Marcos, Facultad de Ciencias Administrativas

\footnotetext{
${ }^{1}$ Doctor en Ciencias Contables y Empresariales por la Universidad Nacional Mayor de San Marcos (UNMSM), Magister en Administración con mención en Gestión Empresarial(UNMSM), Contador Público Colegiado con 38 años de experiencia en la profesión , Profesor Principal de la Facultad de Ciencias Administrativas (UNMSM), ha desempeñado diversos cargos en la Facultad y en entidades del sector público y privado.
}

${ }^{2}$ Jorge Luis Alarcón Cerna: Licenciado en Administración (UNMSM), Maestrista en Educación Superior (UNMSM), Docente (Centro Preparación Universitaria de la UNMSM), Funcionario de Instituciones Públicas.

(C) Los autores. Este artículo es publicado por Gestión en el Tercer Milenio de la Facultad de Ciencias Administrativas de la Universidad Nacional Mayor de San Marcos. Este es un artículo de acceso abierto, distribuido bajo los términos de la licencia Creative Commons Atribucion - No Comercia_Compartir Igual 4.0 Internacional. (http://creativecommons.org/licenses/by-nc-sa/4.0/) que permite el uso no comercial, distribución y reproducción en cualquier medio, siempre que la obra original sea debidamente citada. 


\section{INTRODUCCIÓN}

El estudio que se plantea toma como premisa una realidad socioeconómica existente generalmente en las universidades nacionales, dado en consecuencia deserciones en pleno dictado de clases, motivado, por ejemplo:

- Responsabilidades asumidas no previstas, ocasionando las necesidades de trabajo, distorsionando su atención al desarrollo de los cursos matriculados.

- Enfermedades que la aleja de clases.

- La falta de economía frente a la lejanía del centro universitario y otros; consecuentemente hace que el alumno repita los cursos, según se registra en las diferentes materias, hasta de cuatro repeticiones por curso.

"Frente a esta realidad fáctica nos encontramos con una política pública educativa que deja de ser un instrumento; se encuentra en manos de los poderes nacionales, lográndose un ordenamiento jurídico basada en la superestructura, las relaciones de dominio y de control; con dependencia y sometimiento en las esferas económicas, políticas y culturales" (Rúa Delgado, "Política regulativa en la Educación Superior" (2013).

Por otro lado, se requiere un sistema de aseguramiento de la calidad como una forma de medir intangibles, en los procesos de producción; en el sector educativo debe encontrarse parámetros que permitan una analogía con los procesos de producción. Consecuentemente debe haber una correlación muy directa entre una inversión que produzca utilidad (acción productiva), y la educación.

En ese contexto dentro del ámbito económico internacional, la existencia de clases dominantes de países dependientes aliadas de los capitales transnacionales, derivándose la plusvalía social e intermediario. Luego, como trabajadores son los que desempeñan la labor docente y la materia prima de la producción, los estudiantes como resultado de los procesos productivos.
Que se debe otorgar a la formación permanente de los docentes en ejercicio, como un caso particular de la capacitación profesional;

En el contexto en que nos desarrollamos se generan ideas; pero con sentido y se enriquecen, solo en la experiencia. En la docencia, la experiencia surge, se transforma se reformula en el aula.

Los centros universitarios tienen un papel destacado ante los nuevos retos que plantea la educación permanente. Sus metodologías de formación, las que corresponden a los estudios, deben estar acordes con las exigencias de formación en los avances de la participación, la investigación, y la utilización de recursos multimediables.

Por ello, el estudio que se pretende, es lograr estrategias de resultados en la forma y contenido de la didáctica del docente, frente a una realidad expresada en la formación del alumno. Consideraciones que nos orienta a tomar importancia creciente.

Como antecedentes de nuestra preocupación, podemos señalar:

Rúa Delgado, Carlos Felipe, (2013)," Política Regulativa en la Educación Superior", pg. XVI. presenta un análisis crítico y reflexivo del impacto y las consecuencias de los discursos regulativos en la educación superior y la prospectiva para el siglo XXI, principalmente en lo que atañe a las condiciones geopolíticas en los que se desenvuelve América Latina. El autor nos lleva a lo largo de su ensayo a describir los elementos que de gran manera hicieron que la educación superior, hoy por hoy, atienda básicamente las necesidades de los mercados.

Civarolo, María (2016), "Construyendo la buena enseñanza en la Educación Superior “,pg. 64; nos expresa: "El marco teórico fue construido a partir de la Teoría de la Enseñanza Comprensiva, la Teoría de los Puntos de Acceso, de las Inteligencias Múltiples de Gardner, y de Desarrollo no Universal de Feldman, el Modelo Pedagógico de Dialogante de Zubiria Samper y su vinculación con la Teoría de Vigotski, la noción de Escucha Documentación de Malaguzzi, y la idea de Aula Diversificada de 
Tomlinson, entre otras, ya que todas ellas ofrecen referencias imprescindibles para hacer posible la enseñanza en la diversidad y tomar decisiones para transformar el aula universitaria en una cultura de pensamiento. Concepto acuñado por Perking y su equipo, que supone el trabajo didáctico que busca favorecer la enculturación, proceso que tiene por objetivo pensar y concretar un aula donde se respire el pensamiento.

Pogré Paula (2016)," Enseñar en la Universidad: Una Oportunidad para el Trabajo Reflexivo y en Colaboración", Pg. 185; Trata de empezar a entender que enseñar en la universidad implica poner en dialogo ese sabor específico, disciplinar, que se organiza, se repiensa y se modifica a la hora de su enseñado.

"que, si enseñamos, los estudiantes pueden aprender, nos hace repensar no solo la organización de la enseñanza sino la propuesta pedagógica y colaborativa al interior del trabajo universitario y de las cátedras".

Collazos, Mercedes, (2016)," La Formación Docente para la Educación Superior: El caso de la Universidad Pública Uruguaya", pg171; plantea tres grandes estrategias de formación y perfeccionamiento pedagógico - didáctico:

\section{Actividades de formación perfeccionamiento.}

2. Innovación educativa.

3. Investigación educativa.

Lográndose conformar equipos de formación docente especializada en los diversos campos y especialidades.

En cuanto a la innovación educativa se impulsa a través de mecanismos de proyectos concursales, los que contaría con las unidades pedagógicas de las facultades para el desarrollo descentralizado.

En cuanto a la investigación educativa, menciona dos tipos de acciones:

- Una Maestría en Enseñanza Universitaria.

- Proyectos de Investigación para la mejora de la calidad de la Enseñanza Universitaria.
Alanís Huerta, Antonio, (2002), "El Saber Hacer en la Profesión Docente”, Pg. 79; sostiene "Hoy día, la profesión docente ha dejado de ser un apostolado; ahora es considerado como cualquier otra profesión con exigencias de competencias teóricas, técnicas y contextuales propias del mercado del empleo. Si bien la profesión docente aun no es una profesión liberal, también está sujeta a la competitividad y a las exigencias del contexto social".

\section{MÉTODOS}

\section{Filosofía de la investigación}

Esta investigación es de tipo empírica por ser un hecho que ocurre en la sociedad, es aplicada porque enfrenta el problema, proponiendo alternativas de solución.

El nivel de la investigación es explicativo, se busca las causales que explican el problema en investigación. El diseño es transaccionalcorrelacional. Transaccional, porque el objeto de estudio puede ser analizado en periodos determinados de tiempo.

En concordancia con MARIO BUNGE (Epistemología-1980-Editorial-Arie3lBarcelona), el trabajo se enmarcaría dentro del conocimiento previo que presupone la investigación científica mencionada, en la que constata la validez, la existencia y cognoscibilidad del mundo externo; nuestro estudio está dentro de la esfera técnico y moral al enfocar la "problemática", así como netamente objetivo y realista; se establece una metodología que permite lograr procedimientos pertinentes dirigidos por reglas que logren conocimiento objetivo que se puedan justificar teóricamente y empíricamente.

\section{El enfoque de la investigación}

Es del tipo: Abductivo creativo, porque parte de hechos explicados de tipo inductivo, que han permitido esclarecer una realidad (marco teórico); por lo tanto, considerada cierta, se debe lograr un nuevo caso explicativo y plausible, forma de cambio epistémico por medio del cual se pretende nueva posición de los hechos. La explicación que se pretende debe ser simplemente una sugerencia que deberá ser puesta en prueba antes de ponerse como creencia teórica. 


\section{Estrategias de Investigación}

Se encuadra en la experiencia laboral del autor y las teorías que fundamentan su especialidad en el trabajo docente.

\section{Horizonte de tiempo}

Será de tipo transversal, porque se fundamentan en hechos varios, de un periodo de tiempo determinado.

\section{Métodos de colección de datos}

Datos primarios/secundarios; entrevistas; observaciones.

\section{Método de análisis de datos}

Descriptivos y cualitativos, dado que la evaluación se fundamenta en el logro de un diseño conceptual que permita tomar decisiones dentro de un marco del conocimiento, experiencias y vivencias personales (aspectos que implican grados de subjetividad del ser humano).

La concepción general del trabajo, se enmarcaría:

\section{Trasfondo filosófico.-}

Nos encontraremos dentro del contexto de demarción, dado que, lo que se pretende es lograr una propuesta para un acuerdo o convención; ontológico, la labor se fundamentará en una realidad considerada material (objetiva), como gnoseológica, en el que se busca conocimiento (teoría del conocimiento); así mismo, racional conceptual y lógica. porque se expondrán presuposiciones de la investigación teórica en los campos de la ciencia y de la técnica; constituirá la investigación acciones metodológicas, ya que la investigación parte de cuestionar y termina dentro del proceso, encontrando confirmaciones que han permitido llevar a cabo la presente investigación.

\section{El aspecto formal.-}

Se encuadraría dentro de los alcances de tipo lógico para consistente, como una formalización de la dialéctica a racionalizar en el trabajo.

\section{El cuerpo de conocimiento.-}

Está dentro de la naturaleza y alcance de las Ciencias Sociales, dentro de las cuales se encuentran la Ciencias Administrativas, con las Ciencias Contables; constituyendo el fondo del conocimiento: la persona humana (conocimientos, experiencias y vivencias) insertada en el contexto profesional de la dirección empresarial.

\section{PROCEDIMIENTOS: \\ UNIDAD DE ANÁLISIS}

Estuvo constituido por instituciones del medio, en Lima metropolitana. Sin embargo, como no se trata de un estudio que pretenda generalizaciones para todo el universo; sino más bien, para el logro de las encuestas, nos bastó trabajar con una población seleccionada y estratificada, que permita condiciones de desarrollo de nuestros resultados.

Se debe entender que los resultados a que llegue nuestra investigación concuerdan en general con los puntos de vista expuestos usualmente en la literatura. Representan nuestra conceptualización del problema, basado en la bibliografía existente, como en los cambios de ideas con profesionales de la alta dirección, y en nuestra propia experiencia.

La realización de la observación será mediante encuestas, cuestionarios previamente confeccionados, con el objeto de que dicho elementos de juicio cumplan una función de enlace entre los objetivos de la investigación y la realidad de la población observada. Por ello, se realizó a través de preguntas concretas sobre dicha realidad, y por otra parte debe fue capaz de suscitar en los encuestados, respuestas sinceras y claras a cada pregunta, con el objeto de que puedan ser ordenadas y analizadas. La finalidad del cuestionario es obtener información de la población investigada.

Se establecieron y formularon tablas, que expresarán la disposición conjunta y ordenada de las sumas, o totales obtenidos en la tabulación de datos, referente a las categorías o dimensiones de las variables relacionadas entre sí; apoyados con ordenadores 
electrónicos los que permitieron sistematizar los resultados cuantitativos y ofrecer una visión numérica, sintética y global, del asunto que nos ocupa.

\section{POBLACION}

\section{Selección de la muestra.-}

Se tomó como referencia en la población a considerar aplicables a los sujetos en investigación, a profesores profesionales autorizados en el marco direccional del planeamiento en sus respectivas instituciones; así como de la realización, desarrollo y seguimiento de la misma.

Se consideran a 7 Universidades pública en Lima metropolitana, por estar inmersas en el problema planteado.

\section{Nacionales}

1. Universidad Nacional del Callao (UNAC) (Bellavista)

2. Universidad Nacional Mayor de San Marcos (UNMSM) (El Cercado)

3. Universidad José Faustino Sánchez Carrión (UNJFSC) (Huacho

4. Universidad Nacional Agraria La Molina (La Molina)

5. Universidad Nacional de Educación E. Guzmán y Valle (UNE) (Lima)

6. Universidad Nacional de Ingeniería (UNI) (Rimac)

7. Universidad Nacional Federico Villarreal (UNFV)

Para Torres (1999) "la muestra puede ser determinada por sectores (racimos) geográficos, sociales, educativos, económicos etc. Siempre y cuando los elementos del universo tengan características comunes" (pp 187- 188).

Dado que se investiga a todo el conjunto de sujetos (población), será de tipo censal; ya que la población es pequeña, determinada como alcance del estudio, por lo que no es necesario determinar la muestra.

\section{Construcción del instrumento de medición}

Para la validación del constructo, en el área de asistencia técnica se contó con 3 personas de distintos sexos y edades pertenecientes a la docencia universitaria.

Los cuestionarios fueron realizados a los distintos segmentos de docentes y directivos de las empresas, en sus respectivos lugares de trabajo, responsables del manejo estratégico de la didáctica, en sus respectivas instituciones; con una previa solicitud de visita, solicitud de permiso, conocimiento del formato correspondiente.

De esta forma se pudo interiorizar al encuestado en los fines de la presente investigación, quienes solicitaron su plena confidencialidad, dado de otro modo se estaría quebrando Norma pre-existente de tipo laboral y personal.

La encuesta se llevó a cabo en un promedio de 45 a 60 minutos por persona para poder crear ambiente y empatía entre el encuestado y el encuestador ya que además se deseó saber cuál es la importancia de las distintas dimensiones aplicadas en el SPSS.

Los cuestionarios han dependido su confección a la naturaleza de trabajo de cada personaje implicado, utilizado como medida de valor, la tabla Likert, (5 a 1 en forma decreciente).

Efectuada la encuesta se procesaron los datos, determinando las dimensiones de los ítems más representativos y de esta forma confirmar la calidad de la investigación.

Para calcular la fiabilidad de los datos es utilizo el estadístico Alpha de Cronbach (Cronbach, 1951), prueba la consistencia interna de las variables, reflejando el grado de convocatoria de las preguntas relacionadas con la escala. Para valores inferiores a 0,6 se considera una baja fiabilidad. Entre 0.6 y 0.8 es aceptable. Por encima de 0.8 es excelente, indicará una alta homogeneidad y equivalencia de respuesta a todos los ítems a la vez y para todas las encuestas (Díaz Santos, 2003). 


\section{TÉCNICAS DE RECOLECCIÓN DE DATOS}

Aplicando aleatoriamente los instrumentos (cuestionarios) de evaluación a dos docentes que tengan injerencia en el planeamiento de su institución; afirmando con ello, en lo recomendado por Merten.

\section{ANÁLISIS E INTERPRETACIÓN DE LA INFORMACIÓN}

Fases del estudio:

\section{EL PROCESO}

Para evaluar los datos se utilizó: el SPSS, (STATISTICAL PACKAGE SOCIAL SCIENCEPAQUETE ESTADÍSTICO PARA LAS CIENCIAS SOCIALES), validando la fiabilidad, así como los estadísticos descriptivos y de correlación.

\section{Validación del instrumento de medición}

Dentro de la validación del instrumento de medición se debe corroborar la fiabilidad global del instrumento.

En cuanto a la validez temática se buscó mediante el estadístico correlación de Pearson; el coeficiente del modelo de regresión múltiple; tablas de frecuencias, F de Fischer (Análisis de varianza ANOVA); $t$ de student (coeficiente del modelo).

\section{DISCUSIÓN}

\section{Hipótesis General.-}

Lograr estrategias didácticas para la enseñanza en la Educación Superior, coadyuvaran hacia la eficiencia en el logro del conocimiento y aprendizaje del alumno, dentro de políticas públicas de calidad, en un contexto de realidad nacional, permitiendo intensificar la investigación, con el apoyo de recursos didácticos y técnicos.

\section{Primera Hipótesis Específica.-}

Las Políticas que regulan la Educación, deben ser legitimadas apropiadamente en base su calidad, pertinencia, competencia dentro de una política pública nacional que coadyuve la enseñanza en las universidades nacionales.

\section{Segunda Hipótesis Específica.-}

La enseñanza en las universidades nacionales, debe situarse dentro de una realidad nacional en el contexto de formación del docente universitario.

\section{Tercera Hipótesis Específica.-}

La Investigación en la Educación Superior, debe fundamentarse en las experiencias, desafíos, aprendizaje-servicios continuos, como así, de la competencia del docente que hace realidad la enseñanza en el aula.

\section{Cuarta Hipótesis Específica.-}

Los medios didácticos y tecnológicos relacionados con la enseñanza, permiten retos en las Tecnologías Digital Educativa.

Se pudo aplicar las encuestas cuyos resultados se pueden observar los siguientes acápites. Realizada la muestra, se obtuvo un coeficiente de confiabilidad Alfa de Cronbach 0.821 . Valor que denota la confiabilidad del mismo y los estadísticos mencionados arrojaron alto grado de correlación.

\section{CONCLUSIÓNES:}

La eficiencia de las estrategias didácticas aplicadas a las enseñanzas en Educación Superior depende de las perspectivas políticas nacionales que regulan su legitimidad, calidad, pertinencia, y competencia en la cual están inscritas en el entorno interno (realidad nacional), así como del mejoramiento continuo de los docentes universitarios.

Para el logro de un enfoque sistémico de las estrategias didácticas a ser aplicadas, estas se deben tratar en los niveles de perspectivas señalas, evaluando y aplicando el marco teórico pre-existente de la enseñanza en los niveles superiores en las universidades, tener presente al docente universitario comprometido (profesor), en sus logros de investigador, experiencias y vivencias personales, y desafíos; como su aprendizaje continuo, y su aplicación en competencia.

Finalmente, un adecuado proceso de los recursos didácticos y tecnológicos en la Educación Superior, coadyuvaran en la 
aplicación didáctica del profesor en el aula, mediante medios electrónicos y tecnológicos, que permitan retos en el aprendizaje continuo del alumno.

Nuestro estudio trato de resolver la construcción de una estrategia didáctica, teniendo presente la existencia de un conjunto de prácticas que aunque no garantizan que al final se tenga un buen resultado, sirva como marco de referencia para lograr un aprendizaje didáctico realizable; he aquí un plan piloto realizado una de las universidades señaladas

Generalmente en las universidades nacionales existen ocurrencias en las que los profesores tratamos de sobrellevar y mentalizarnos en una solución posible frente a problemáticas como las señaladas en el presente artículo, como también a preguntas que el alumno al iniciar el Ciclo de Enseñanza; como La siguiente: ¿cuál es método de enseñanza?; a lo que el profesor pasó a responder y comentar, lo siguiente:

- Se visualizaran los temas según Syllabus del Curso, en forma semanal; así mismo, se les entregará, en el escritorio del CPU, dos PPTs, el explicado y visualizado por el profesor en clase y otro del mismo tema, pero con distinto enfoque, así mismo se les entregará un marco teórico del tema, de aproximadamente 20 páginas, como lectura y estudio; también en el escritorio, para ser trabajado en grupos de discusión, (no más de cuatro personas), durante una semana; en la próxima clase, se logra por alumno en forma escrita un resumen del tema realizado, hasta 20 líneas de un A4, y diez preguntas sobre el tema estudiado y respuestas, que no pasen de cuatro líneas; durante la clase de presentación de resúmenes y preguntas, se establece una dinámica de interrogantes o vacíos detectados sobre lo realizado por los alumnos; siendo el profesor un coordinador o en todo caso da respuestas y aclaraciones pertinentes a los diferentes casos que se pudieran presentar (lográndose a lo que denomina: Dinámica de Grupos.
- Lo señalado es registrado en los controles del profesor en forma interna y personalizada con el alumno, el objetivo es evaluar el desarrollo continuo y permanente de alumno, registrándose en las Actas; permitirá ser objetivo en las calificaciones a ser realizadas.

La estrategia descrita ha sido durante más de cuatro años, finiquitado con gran éxito en los distintos Ciclos de Estudio con respecto al curso del profesor, con la anuencia voluntaria de alumnos en la aplicación de la estrategia didáctica, sin soslayar los requerimientos y procedimientos de aprendizaje en la propia universidad, pre-existentes; por lo tanto existe Consistencia de lo realizado, sin problema alguno.

Por otro lado, cabe también la realización de Aulas Virtuales, o por Correo Electrónico, con consultas personalizadas periódicamente, para aquellos alumnos que pudieran tener dificultades como las señaladas en nuestra Introducción.

\section{REFERENCIAS BIBLIOGRAFICAS}

Alanis Huerta, Antonio, (2002)," El Saber Hacer en la Profesión Docente". Editorial TRILLAS, México D.F., México.

Bunge Mario (Epistemología -1980-Editorial Ariel31-Barcelona).

Civarolo, María Mercedes (2016), “Construyendo la buena enseñanza en la Educación Superior, Buenos Aires, Argentina; NOVEDUC.

Collazos, Mercedes (2016),"La Formación de Docentes para Superior: El caso de la Universidad Pública uruguaya. Buenos Aires Argentina; NOVEDUC.

Cronbach L (1951) Coeffient Alpha and the Internal Structure of Test. Psycometrika (setiembre) pp 297-334.

Díaz Santos, Sep 3, 203-368 pg. Estadística Aplicada: Una visión instrumental.

Fernández Barcala M. (2000),"Validación de Serqual como instrumento de medida de calidad de servicio bancario. Revista Europea de Dirección y Economía de la Empresa, volumen 9, No. Pp 57-70.

Pogré, Paula (2016)"Enseñar en la Universidad: Una oportunidad para el trabajo reflexivo 
y en colaboración "Buenos Aires Argentina; VOVEDUC.

Rua Delgado, Carlos Felipe (2013),"Política Regulativa en la Educación Superior",1ra edición, Editorial ECOE, Bogotá.

Torres Manzanera - Datos del profesor - Universidad de Oviedo. https://directo.uniovi.es/catalogo/ DetalleProfesor.asp?idprofesor
Visouta, Análisis estadístico con SPSS para Windows: estadística. https://www. researchgate.net/.../31753773_Analisis_ estadistico_con_SPSS_para_Windows estadistica_multivariable_B_Visauta_Vinacua_ JC_Martori 